\title{
Coherent X-Ray Diffraction Imaging of Morphology and Strain in Nanomaterials
}

\author{
ROSS HARDER ${ }^{1,4}$ and IAN K. ROBINSON ${ }^{2,3}$ \\ 1.--Advanced Photon Source, Argonne National Laboratory, Argonne, IL 60439, USA. 2.-London \\ Centre for Nanotechnology, University College London, London WC1E 6BT, UK. 3.-Research \\ Complex at Harwell, Didcot, Oxfordshire OX11 0DE, UK. 4.-e-mail: rharder@aps.anl.gov
}

The last decade has seen a remarkable surge in x-ray characterization methods (Willmott, An Introduction to Synchrotron Radiation, John Wiley \& Sons, Inc., New York, 2011). Imaging with x-rays has evolved from simple radiography, to image internal structure and diagnose injury, to a full-fledged tool for nanoscale characterization (Holt et al., Annu Rev Mater Res 43:1, 2013). Central to this development has been the advent of high-brilliance synchrotron and free electron laser sources of x-rays. The high degree of spacial coherence of the resulting beams has enabled novel imaging methods. Of these, coherent diffraction imaging has proven highly successful at imaging the structure in nano materials (Miao et al., Nature 400:342, 1999). In addition, this imaging method can be combined with Bragg diffraction to image strain with high sensitivity (Pfeifer et al., Nature 442:63, 2006; Robinson and Harder, Nat Mater 8:291, 2009).

\section{INTRODUCTION}

Since the discovery of x-rays by Röntgen in $1895^{1}$ the penetrating power and short wavelength of this form of light has been exploited in many ways. ${ }^{2}$ Indeed, the term "x-ray" has even become synonymous with the radiography images that are commonplace in medicine to noninvasively diagnose injury and disease. Perhaps less common in everyday experience is the concept of diffraction of $x$-rays by periodic structures such a crystals. This phenomenon was discovered by the father-son team of Bragg and Bragg in $1913^{3,4}$ and has contributed tremendously to society, even though one seldom encounters Bragg's diffraction spots on a daily basis.

Bragg diffraction arises due to the extremely short wavelength of x-ray light. X-rays have wavelengths similar to the spacing between atomic planes within a crystal. It is possible to orient a crystal in such a way that x-ray beams reflected from individual lattice planes can constructively add up in relative phase, via exactly the same mechanism that gives rise to maxima and minima in the classic Young's double slit experiment. With such an alignment, one can observe extremely bright, reflected x-ray beams (Bragg beams) from specific orientations of the crystal lattice. When the alignment condition is not carefully met, the incident beam will pass through the sample or be simply absorbed in the form of heat. This behavior is fundamentally different than reflection of visible light by a mirror, as that reflected intensity is relatively insensitive to the angle. The very nature of Bragg diffraction means that it has exquisite sensitivity to the atomic structure of matter. It is this powerful capability that has allowed the determination of the atomic structure of everything from the genetic building blocks of life ${ }^{5}$ to advanced materials for efficient lighting. ${ }^{6}$

Radiography and diffraction are in some sense the opposite ends of the spectrum for structural studies of matter. Although the diffraction condition gives average atomic scale information of the sample, radiography gives images of local structure in the micro-millimeter length scales. Quite often the local structure has a profound impact on the properties of the material. This is particularly evident within nanomaterials, or objects having spacial dimensions of a few hundreds of nanometers or less. As pointed out by Robinson et al., ${ }^{7}$ the unique properties of these materials require innovative new tools to study them, especially when one needs to study these materials in their native setting or within complex or corrosive environment. 
To study the local structure of materials at spatial resolution better than $1 \mathrm{um}$, the x-ray equivalent of a microscope is a powerful tool. ${ }^{8}$ The foundation of high-resolution x-ray microscopy is the x-ray Fresnel zone plate (FZP) lens. Due to the weak interaction of the x-rays with matter, a diffractive optic like an FZP is ideal. Currently the best FZPs designed for hard x-ray (short wavelength) use are reaching down to $20-\mathrm{nm}$ resolution. ${ }^{9}$ There are substantial technical challenges in producing diffractive lenses with smaller features sizes and hence higher resolution. Actually, the current record for focusing hard x-rays goes to a mirror system that produced a 7-nm focused x-ray beam. ${ }^{10}$ Both mirror and diffractive $\mathrm{x}$-ray lenses are technologically challenging, and as with optical microscopy, high resolution comes at the cost of the working distance of the lens. In the case of x-ray microscopy, this cost is quite high as one of the greatest benefits of hard x-rays, in particular, is their greater penetration into both materials and environmental cells that are used to expose samples to high or low temperatures, toxic gases, or extreme pressures, for example. ${ }^{11,12}$

When one wishes to study a sample within an environmental cell, or needs information at resolutions beyond that of the best x-ray lenes, the lens of the microscope can be replaced with computational algorithms. This method, known as coherent diffractive imaging (CDI), exploits the brilliance of modern synchrotrons and free electron lasers ${ }^{13}$ to image materials at potentially very high spatial, ${ }^{14,15}$ and now temporal, resolution. ${ }^{16,17}$ The reason the method can image the local structure, even without a lens, is that the beam itself has a point-to-point phase correlation that is preserved throughout the interaction with the sample. The x-rays scattered from different regions of the sample reach a camera with a fixed relative phase to each other and constructively or destructively interfere accordingly. So although not formally identical to the diffraction from crystal planes that Bragg originally described, the fact that interference of waves scattered from different regions of the sample is what is measured makes the name appropriate. Essentially the squared modulus of the Fourier transform of the structure of the sample is measured at the detector plane. As a result, CDI is subject to an obstacle similar to the phase problem of x-ray crystallography. ${ }^{18,19}$ The key to producing an image from the measured data, which bears little obvious resemblance to the sample, is to computationally retrieve the phases of the scattered waves and propagate, via Fourier transform, back to the plane of the sample. ${ }^{20-22}$

The resolution of CDI is determined in a similar way to lens-based microscopy. Although the microscope resolution is determined by the numerical aperture (NA) of the lens, the CDI resolution is determined by the numerical aperture of the data, or the largest angle that the x-rays are scattered by the sample. In the language of diffraction, it is the maximum momentum transfer imparted to the scattered beam that determines the resolution of the data and, hence, the resolution of the obtained image. As a result of the need to scatter x-rays to greater angles to obtain greater resolution, materials with greater electron density (high-z) like metals and some semiconductors can be imaged more easily at greater resolution. Low-z materials like silicon and carbon, for instance, scatter much less and hence must be exposed to the x-ray beam for a much greater measurement period to obtain highresolution images.

The dominant scattering mechanism, giving rise to image contrast, is the electron density. Coherent imaging can possesses remarkable sensitivity to material properties. In Diaz et al. ${ }^{23}$ they illustrate a $2 \%$ accuracy in determination of the density of $\mathrm{SiO}_{2}$ microspheres. Coherent imaging is being developed to exploit a variety of other contrast mechanisms. Linear dichroism in magnetic systems has already been used to image labyrinth domains in GdFe films ${ }^{24}$ and circular dichroism with soft x-rays has been used to study the magnetic structure in $\mathrm{CoPt}$ films using a form of holography. ${ }^{25}$ Instrumentation to exploit circular magnetic dichroism as an imaging contrast with hard x-rays is currently being developed. In addition, coherent imaging can be combined with the exquisite sensitivity of $\mathrm{x}$-rays to distortions of a crystalline lattice to image strain. Usually referred to as Bragg coherent diffractive imaging (BCDI), the ability to image strain on the nanometer scale in three dimensions is highly novel. ${ }^{26,7}$

Coherent imaging is currently done in two somewhat distinct modes. The original method relies on the sample being compact in space or smaller than the coherent x-ray beam in which it is measured. ${ }^{27,20}$ The measurement can be done either in the small angle geometry or in the vicinity of Bragg peaks of crystals. In the former case, the scattered $\mathrm{x}$-rays are near the direct beam, while in the latter, the sample must be a crystal and the detector and sample are oriented at a Bragg condition of the lattice.

The most recent addition to coherent imaging methods has been generally called ptychography or scanning $x$-ray diffractive imaging. ${ }^{28,29}$ The goal of this method is to remove the requirement that the sample be compact. Ptychography can image, with all of the benefits of greater working distance and potentially higher resolution, an extended field of view in a sample of larger size. The root of the word "ptychography" is from the Greek meaning "to fold," and that is the principle behind the method. The phase retrieval process of CDI is not very robust when the object is not compact or finite in extent. To overcome this technical difficulty, the beam is scanned across the sample and measurements are done at overlapping locations. This folding of the measurements from overlapping regions of the sample greatly aids in the phase retrieval process to produce the image of the sample. 
In recent years, the phase retrieval algorithms have been the greatest area of development in the field. For CDI, they are still primarily based on the iterative algorithms of Gerchberg and Saxton, which were developed for the case of two measurements. ${ }^{30}$ One done in diffraction space, identical to CDI, and another done in direct, or image, space, where with an electron microscope one can switch at the push of a button. The algorithms are iterative in nature, using a computational tool known as the fast Fourier transform (FFT) to switch back and forth between the two measurement spaces. Although the FFT is a topic of research on its own, there are canned libraries that are reasonably robust. In the Gerchberg and Saxton case, they were attempting to improve the resolution of the images formed in an electron microscope. In the CDI case, we have only one measurement in the diffraction space. The algorithms coined hybrid input-output (HIO) and error reductions (ERs) were defined in Fienup's 1982 paper and are the true workhorses of CDI. ${ }^{20}$ For CDI, as there is no measurement in direct space, we must encode as much information as we have regarding the sample to aide the phase retrieval algorithm in convergence. Knowledge of the size of the sample is encoded in a compact support constraint that must include the space occupied by the sample. This support, or space for the image to form in the computational array, determines how the electron density of the image is modified at each iteration. A formulation by Marchesini et al. ${ }^{31}$ called shrinkwrap allows for the support size to be automatically determined as the iterations of the algorithm proceed. Chen et al. developed a method to improve the reliability of an image called guidedHIO (GHIO). In GHIO, multiple sequences of phase retrieval are started from random distributions and the resulting images from each are combined together periodically during the iterations, in a variety of ways, to converge to a single solution. ${ }^{32}$ One of the most recent additions to the iterative algorithms has been the ability to correct for imperfect coherence in the x-ray beam. ${ }^{22}$ This can arise as a consequence of the beam line configuration (slits opened too wide) or scatter from windows that the x-ray beam must pass through. Poor coherence in the beam reduces the contrast in the bright and dark regions of the diffraction pattern. In direct space, the density recovered will typically be less uniform. This method was critical for application of BCDI to a sample within a high-pressure diamond anvil cell. ${ }^{33}$ Due to the presence of a gasket that was traversed by the beam both entering and exiting the cell, the partial coherence of the incident beam became more severely intertwined. ${ }^{12}$

In this text, we describe an example of coherent imaging of a nanometer-scale crystal of barium titanate mounted on a thick silicon substrate. In this example, the compact nature of the sample is taken into account during the phase retrieval process to produce an image. Since this measurement

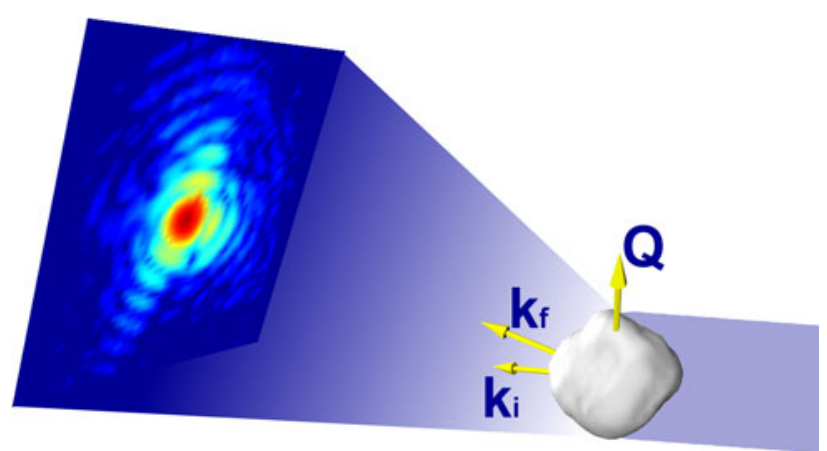

Fig. 1. Illustration of the BCDI experiment at APS 34-ID-C. The 320$\mathrm{nm}$-diameter barium titanate crystal is aligned at the 110 Bragg angle at the center of the diffractometer. A slice through the three-dimensional coherent diffraction pattern is measured in the far field at the Bragg angle indicated by the direction of the arrow labeled $\mathbf{k}_{\mathbf{f}}$. The difference between the incident momentum vector $\mathbf{k}_{\mathbf{i}}$ and $\mathbf{k}_{\mathbf{f}}$ is given by the arrow labeled $\mathbf{Q}$. It is the direction of $\mathbf{Q}$ onto which the distortions of the crystal lattice are projected in our BCDI images and is the so-called reciprocal lattice vector that defines the Bragg condition.

was done in the vicinity of a (110) Bragg peak of the crystal, we were sensitive to distortions of the lattice due to strain. Intuitively the strain sensitivity comes from the fact that different positions in the crystal will have slightly different lattice spacing and hence diffract x-rays to minutely different angles. Due to the coherence of the beam, this fact will be encoded in the interference pattern produced in the near vicinity of the Bragg peak. By the nature of Fourier transforms, upon computational phase retrieval, the image will be of a complex (containing both real and imaginary parts) density distribution within the object. The amplitude of the complex numbers is directly proportional to the electron density of the crystal, while the phase of the complex numbers is directly related to the distortion of the lattice along the direction of the crystal planes that are aligned at the Bragg condition.

Figure 1 illustrates the BCDI experiment at beamline 34-ID-C of the Advanced Photon Source (APS) at Argonne National Laboratory. At this synchrotron beamline, the coherent fraction of the synchrotron source is our primary concern. Precision slits are almost the only optical components that touch the beam. The instrument does contain a monochromator, which is a pair of silicon (111) oriented crystals, to select just a single wavelength of x-rays from the spectrum produced by the synchrotron. These crystals are carefully polished to maintain the purity of the coherent wavefront. After the monochromator, there is a precision slit that selects just a single coherent mode from the beam and a Kirk-Patrick Baez mirror system that focuses the x-ray beam from tens of micrometers down to less than $1 \mu \mathrm{m} .{ }^{34}$ Again, this focusing optic does not determine the final resolution of the image; it is simply used to concentrate as much of the coherent beam onto a tiny sample as possible. This focusing 
system has a long working distance, in x-ray lens terms, of $7 \mathrm{~cm}$ or more, leaving room for temperature stages, gas environments, high-pressure cells, or any other in situ action the scientist can conceive of.

\section{MORPHOLOGY AND STRAIN IN BTO NANOCRYSTALS}

BCDI has been used to study nanosized barium titanate (BTO) at APS. BTO's dielectric constant peaks as a function of size with a maximum of around $140 \mathrm{~nm}$ with a factor of 3 enhancement. This property makes nanoscale BTO an important industrial material for making supercapacitors. ${ }^{35}$ As energy storage is a major component of renewable energy technologies, materials such as this are of great interest to this major industry.

The structural origin of the dielectric enhancement in nanometer-sizes crystals of BTO is an important scientific question that can be addressed by BCDI. The dielectric susceptibility, the amount of electric polarization for a given applied field, is a structural quantity related to the degree of ionic displacements allowed in a given lattice. The ions are expected to have fixed charges, so the polarization is directly proportional to the lattice distortion. Nanoparticles have a large surface-to-volume ratio so they can presumably take advantage of additional degrees of structural freedom at their surfaces, which in turn allows for a higher polarization. The question of how the electric polarization is distributed within the nanoparticle demands threedimensional spatial imaging of the crystals distortions, as BCDI can provide.

BTO has a classic perovskite structure, for which the parent phase (above $100^{\circ} \mathrm{C}$ at ambient pressure) is cubic, becoming tetragonal at room temperature. $^{36}$ This phase transition is influenced by pressure and by particle size, which results in effective pressure. ${ }^{37}$ The dielectric polarization, which results from oxygen ion displacements in the opposite direction from the $\mathrm{Ba}$ and $\mathrm{Ti}$ cations, is present in all phases. ${ }^{38} \mathrm{~A}$ recent publication used x-ray powder diffraction analysis to infer a core-shell structure for the nanocrystalline BTO. ${ }^{39}$ The experiments used Rietveld refinement to fit the splitting of the Bragg peaks observed for a range of particle sizes to determine the ratio of tetragonal and cubic material in the sample. The core-shell picture, in which the core is tetragonal and the shell is cubic, was proposed to explain the size-dependence of those ratios. This core-shell model, which could explain the enhancement of dielectric constant, can be imaged directly by BCDI without the need to invoke models.

BCDI is a well-established technique at the $\mathrm{APS}^{7}$ that is able to image nanometer-sized crystals in $3 D$ with a resolution of $30 \mathrm{~nm}$ or better. Strains are detected as phase in the direct-space images and can be quantitatively analyzed as projections of the local displacements onto the scattering $\mathrm{Q}$-vector. The tetragonal/cubic core shell structure would therefore show up as a broadening of the cubic-index Bragg peaks, translating into characteristic phase patterns of the $3 \mathrm{D}$ images of the crystals. We have previously observed core-shell strain structures on micron-sized zeolite crystals. ${ }^{40}$

BCDI has been found to be effective in looking at twinning in complex oxide crystals. ${ }^{41}$ Merohedrally twinned crystals have two (or more) orientations of crystal grains that are joined at a common interface located at one of the crystallographic planes. The crystallographic description is complicated in general by the presence of two lattices, but the generalized phase approximation $(\mathrm{GPA})^{42}$ can be used to represent one lattice as a distortion of the other. ${ }^{38}$ The second crystal is considered to have complex electron density in which its local orientation is represented as a phase at every point in space. Using the GPA, the second crystal acquires a phase ramp when described in the coordinate system of the first crystal of the twin. ${ }^{41}$ In this way, the tetragonal-cubic, core-shell structure proposed by Hoshina ${ }^{39}$ will be identified as a flat phase in the interior (tetragonal reference lattice) with phase ramp structures on the outside representing the cubic crystallographic phase, which appears as a distortion.

In this case, the sample consists of a silicon wafer that has been sprinkled with BTO nanocrystals. The sample was mounted at the center of the diffractometer at beamline 34-ID-C of APS, and the detector was placed at the nominal Bragg angle for BTO (110). Since many hundreds of crystals are in the x-ray beam at any given position of the sample, it was quite easy to find a Bragg diffraction beam from an individual nanocrystal like the one shown in Fig. 1. To measure the entire 3D coherent diffraction pattern in the vicinity of the Bragg beam, the crystal is rotated in direct space to translate the 3D coherent diffraction pattern in reciprocal space through the area detector. A typical measurement of a coherent diffraction pattern from crystals of this size will involve 50 or more angles within one half to one degree of the Bragg angle. The individual slices of the 3D diffraction pattern are then stacked together to be fed into the phase retrieval programs.

We investigated the structures of $320-\mathrm{nm}$ and 240-nm BTO crystals using BCDI. The diffraction patterns in Fig. 1 show a clear fringed diffraction pattern from the facets of the $320-n m$ crystal. Images of the 320-nm crystal in Fig. $2 \mathrm{a}$ and $\mathrm{b}$ show strains localized at two of the facets of the crystal shape. Figure 2c shows a slice through the phase of the three-dimensional density. The slice shows an interior that is a relative flat phase, except for one small region where the density appears missing. The opposite shade phases on either size of this point indicate the possible presence of a defect in the lattice that induces a dipole-like distortion field. The density here is not actually missing, but the lattice is sufficiently distorted that some of the phase 


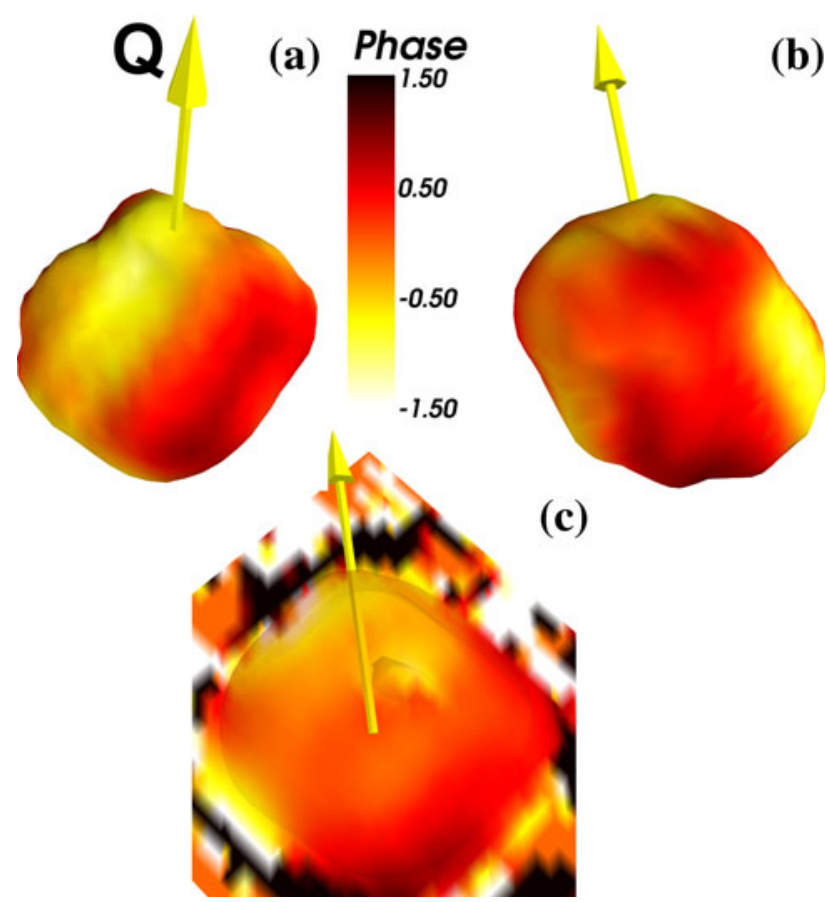

Fig. 2. ( $a$ and b) Views of opposite sides of the 320-nm-diameter BTO crystal shown in Fig. 1 produced from the tetragonal-sensitive 110 Bragg peak. The three-dimensional contour, or isosurface, is at the $25 \%$ level. The shading on the surface is determined by the phase of the density, or distortion of the lattice, at a given position. The total range in phase, from -1.5 to +1.5 radians, corresponds to approximately one half of the 110 lattice spacing, or 2.8 angstroms of accumulated displacement along the direction indicated by the $\mathbf{Q}$ vector in the illustration. (c) The phase of the interior is seen on the slice through the phase of the 3D volume. The randomized phases outside of the semitransparent isosurface can be disregarded.

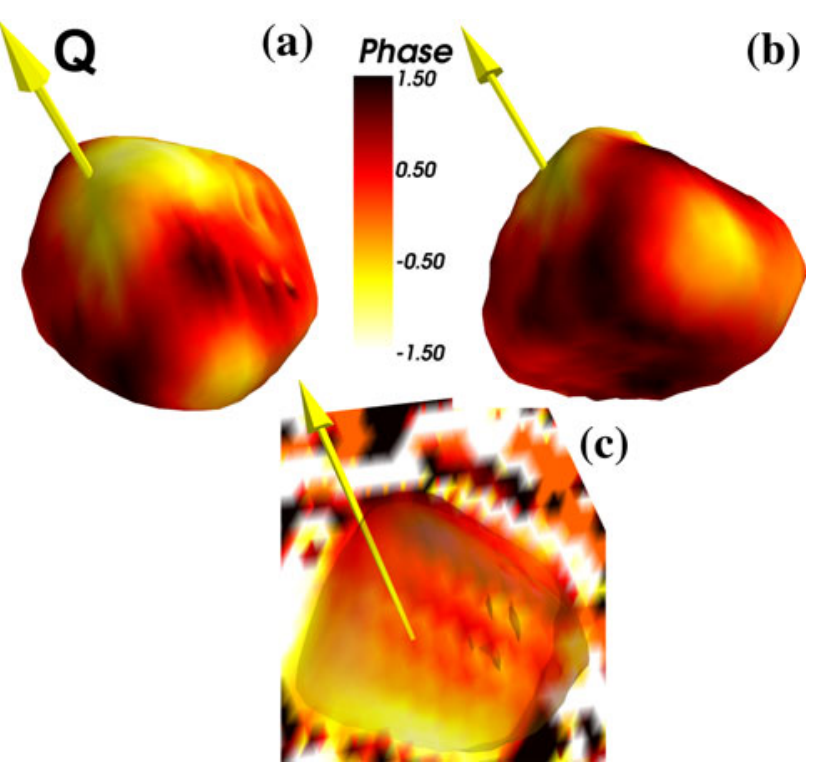

Fig. 3. ( $a$ and $b$ ) Views showing the top and bottom of a phaseisosurface of another BTO crystal with a diameter of about $240 \mathrm{~nm}$. The arrow indicates the direction of the reciprocal lattice vector onto which the distortion of the lattice is projected. (c) A slice through the phase of the complex image is shown cutting a semitransparent isosurface in the amplitude. The phase of the interior is relatively flat relative to the outer edges where dark and light regions are obvious. contrast in direct space has caused interference and has reduced the amplitude of the complex density. In Fig. 3, a similar set of images are shown of the 240-nm crystal. The phase-isosurface images show greatest strains on the top and bottom of the crystal, relative to the $\mathrm{Q}$ vector of the 110 Bragg peak. As in the larger crystal, the interior is a relatively flat phase. There is an obvious scallop pattern shown in the phase slice, but this is likely a structure below the resolution of the image and hence not reliable. These images indicate a pattern of strain in the particles that might be consistent with a core-shell strain model where the interior is relatively unstrained and the lattice in the vicinity of the surfaces is more so.

\section{SUMMARY}

CDI is rapidly establishing itself at x-ray light sources like the APS at Argonne National Laboratory and the Linac Coherent Light Source at the Stanford Linear Accelerator, both of which are examples of National User Facilities (www.nufo. org). The imaging method is highly dependent on these high brilliance sources to produce the coherent beams. Dedicated instrumentation exists at these facilities and is supported by professional staff to run experiments with visitors. The reader should be aware that these capabilities are available to scientists with all levels of x-ray imaging background. Generally the national user facilities operate through a proposal review process that scores and prioritizes experiments to be done at each of the instruments. For the novice user, the instrument staff are generally very helpful in all stages of the proposal process and all are encouraged to engage these facilities in their research.

\section{ACKNOWLEDGEMENTS}

This work is supported by the U.S. Department of Energy, Basic Energy Sciences, Office of Science, under Contract DE-AC02-06CH11357.

\section{REFERENCES}

1. W.K. Röntgen, Science. New Series 3, 726 (1896).

2. P. Willmott, An Introduction to Synchrotron Radiation (New York: John Wiley \& Sons, Inc., 2011), pp. i-xv.

3. W.H. Bragg and W.L. Bragg, Proc. R. Soc. Lond. Ser. A 88, 428 (1913).

4. W.L. Bragg, Proc. R. Soc. Lond. Ser. A 89, 248 (1913).

5. J.D. Watson and F.H.C. Crick, Nature 171, 737 (1953).

6. H. Amano, N. Sawaki, I. Akasaki, and Y. Toyoda, Appl. Phys. Lett. 48, 353 (1986).

7. I. Robinson and R. Harder, Nat. Mater. 8, 291 (2009).

8. Y.-T. Chen, T.-N. Lo, Y.S. Chu, J. Yi, C.-J. Liu, J.-Y. Wang, C.-L. Wang, C.-W. Chiu, T.-E. Hua, Y. Hwu, Q. Shen, G.-C. Yin, K.S. Liang, H.-M. Lin, J.H. Je, and G. Margaritondo, Nanotechnology 19, 395302 (2008).

9. J. Vila-Comamala, Y. Pan, J.J. Lombardo, W.M. Harris, W.K.S. Chiu, C. David, and Y. Wang, J. Synchrotron Radiat. 19, 705 (2012).

10. H. Mimura, S. Handa, T. Kimura, H. Yumoto, D. Yamakawa, H. Yokoyama, S. Matsuyama, K. Inagaki, K. Yamamura, Y. Sano, K. Tamasaku, Y. Nishino, M. Yabashi, T. Ishikawa, and K. Yamauchi, Nat. Phys. 6, 122 (2009). 
11. M. Holt, R. Harder, R. Winarski, and V. Rose, Annu. Rev. Mater. Res. 43, 1 (2013).

12. W. Yang, X. Huang, R. Harder, J.N. Clark, I.K. Robinson, and H.-K. Mao, Nat. Commun. 4, 1680 (2013).

13. I.A. Vartanyants and A. Singer, New J. Phys. 12, 035004 (2010).

14. Y. Takahashi, N. Zettsu, Y. Nishino, R. Tsutsumi, E. Matsubara, T. Ishikawa, and K. Yamauchi, Nano Lett. 10, 1922 (2010).

15. Y. Takahashi, Y. Nishino, R. Tsutsumi, H. Kubo, H. Furukawa, H. Mimura, S. Matsuyama, N. Zettsu, E. Matsubara, T. Ishikawa, and K. Yamauchi, Phys. Rev. B 80, 054103 (2009).

16. K. Gaffney and H. Chapman, Science 316, 1444 (2007).

17. J.N. Clark, L. Beitra, G. Xiong, A. Higginbotham, D.M. Fritz, H.T. Lemke, D. Zhu, M. Chollet, G.J. Williams, M. Messerschmidt, B. Abbey, R.J. Harder, A.M. Korsunsky, J.S. Wark, and I.K. Robinson, Science 341 (6141), 56 (2013).

18. D. Sayre, Acta Cryst. 5, 60 (1952).

19. J. Karle and H. Hauptman, Acta Cryst. 3, 181 (1950).

20. J. Fienup, Appl. Optic. 21, 2758 (1982).

21. R. Harder, M. Liang, Y. Sun, Y. Xia, and I.K. Robinson, New J. Phys. 12, 035019 (2010).

22. J.N. Clark, X. Huang, R. Harder, and I.K. Robinson, Nat. Commun. 3, 993 (2012).

23. A. Diaz, P. Trtik, M. Guizar-Sicairos, A. Menzel, P. Thibault, and O. Bunk, Phys. Rev. B 85, 020104 (2012).

24. A. Tripathi, J. Mohanty, S.H. Dietze, O.G. Shpyrko, E. Shipton, E.E. Fullerton, S.S. Kim, and I. McNulty, Proc. Natl. Acad. Sci. 108, 13393 (2011).

25. S. Eisebitt, J. Lüning, W. Schlotter, and M. Lörgen, Nature 32,885 (2004).

26. M.A. Pfeifer, G.J. Williams, I.A. Vartanyants, R. Harder, and I.K. Robinson, Nature 442, 63 (2006).

27. J. Miao, P. Charalambous, J. Kirz, and D. Sayre, Nature 400, 342 (1999).
28. J. Rodenburg, A. Hurst, A. Cullis, B. Dobson, F. Pfeiffer, O. Bunk, C. David, K. Jefimovs, and I. Johnson, Phys. Rev. Lett. 98, 034801 (2007).

29. P. Thibault, M. Dierolf, A. Menzel, O. Bunk, C. David, and F. Pfeiffer, Science 321, 379 (2008).

30. R.W. Gerchberg and W.O. Saxton, Optik 35, 237 (1972).

31. S. Marchesini, H. He, H. Chapman, S. Hau-Riege, A. Noy, M. Howells, U. Weierstall, and J. Spence, Phys. Rev. B 68, 140101 (2003).

32. C.-C. Chen, J. Miao, C.-L. Wang, and T.-K. Lee, Phys. Rev. B 76, $064113(2007)$

33. H.-K. Mao, J. Xu, V.V. Struzhkin, J. Shu, R.J. Hemley, W. Sturhahn, M.Y. Hu, E.E. Alp, L. Vocadlo, D. Alfe, G.D. Price, M.J. Gillan, M. Schwoerer-Boihning, D. Hiäusermann, P. Eng, G. Shen, H. Giefers, R. Lüibbers, and G. Wortmann, Science 292, 914 (2001).

34. M.R. Howells, D. Cambie, R.M. Duarte, S. Irick, A.A. MacDowell, H.A. Padmore, T.R. Renner, S. Rah, and R. Sandler, Opt. Eng. 39, 2748 (2000).

35. G. Arlt, D. Hennings, and G. de With, J. Appl. Phys. 58, 1619 (1985).

36. G. Shirane and K. Suzuki, J. Phys. Soc. Jpn. 6, 274 (1951).

37. I. Robinson, J. Phys. Soc. Japan 82, 021012 (2012).

38. G. Shirane, B. Frazer, V. Minkiewicz, J. Leake, and A. Linz, Phys. Rev. Lett. 19, 234 (1967).

39. T. Hoshina, S. Wada, Y. Kuroiwa, and T. Tsurumi, Appl. Phys. Lett. 93, 192914 (2008).

40. W. Cha, N.C. Jeong, S. Song, H.-J. Park, T.C. Thanh Pham, R. Harder, B. Lim, G. Xiong, D. Ahn, I. McNulty, J. Kim, K.B. Yoon, I.K. Robinson, and H. Kim, Nat. Mater. 12 (8), 729 (2013)

41. M.A.G. Aranda, F. Berenguer, R.J. Bean, X. Shi, G. Xiong, S.P. Collins, C. Nave, and I.K. Robinson, J. Synchrotron Radiat. 17, 751 (2010).

42. M. Hÿtch, F. Houdellier, F. Hüe, and E. Snoeck, Nature 453, 1086 (2008). 